\title{
Index of Objects
}

\begin{tabular}{|c|c|c|c|}
\hline $0913+391$ & 106 & $\beta$ Aur & 412,414 \\
\hline $0920+390$ & 446 & $\beta$ Cep & 412 \\
\hline $0923+392$ & 106 & $\beta \mathrm{CMi}$ & 408 \\
\hline $1038+528 \mathrm{~A}, \mathrm{~B}$ & 446 & $\beta$ Gru & 328 \\
\hline $113 \mathrm{Her}$ & 412 & $\beta$ Per & 412,414 \\
\hline 12 Per & 412 & $\beta$ Tri & 412 \\
\hline $1222+037$ & 106 & Cas A & 245,337 \\
\hline $1226+023$ & 106 & Cen A & 132 \\
\hline $1422+231$ & 129 & Ceres & 279 \\
\hline $1424+240$ & 129 & $\chi$ Cyg & 386,396 \\
\hline $1611+343$ & 106 & $\chi$ Dra & 412 \\
\hline $1638+398$ & 106 & Cyg A & $245,337,352$ \\
\hline $1641+399$ & 106 & $\delta$ Cap & 347 \\
\hline $1641+399$ & 106 & $\delta \operatorname{Tri}$ & 412 \\
\hline $1803+784$ & 446 & DG Tau & 417 \\
\hline $1928+738$ & 446 & DL Tau & 417 \\
\hline $1934-638$ & 355 & $\mathrm{El} 30$ & 417 \\
\hline $2007+777$ & 446 & EL 38 & 417 \\
\hline $2311-452$ & 355 & El 44 & 417 \\
\hline $30 \mathrm{Her}$ & 396 & $\epsilon$ Aur & 414 \\
\hline 32 Boo & 346 & $\eta$ And & 412 \\
\hline $3 \mathrm{C} 309.1$ & 368 & $\eta \mathrm{Car}$ & 279 \\
\hline $3 \mathrm{C} 345$ & 446 & $\eta \mathrm{Oph}$ & 347 \\
\hline $3 \mathrm{C} 382$ & 446 & $\eta$ Peg & 412 \\
\hline $3 \mathrm{C} 395$ & 446 & $\eta \mathrm{Tau}$ & 408 \\
\hline $48 \mathrm{Per}$ & 408 & FV Tau & 417 \\
\hline $4 \mathrm{C} 31.04$ & 126 & FW Tau & 417 \\
\hline $4 C 39.25$ & 446 & FY Tau & 417 \\
\hline $6 \mathrm{Lac}$ & 412 & $\mathrm{G} 78.2+2.1(2000+412)$ & 353 \\
\hline AA Tau & 417 & Galactic centre & 379 \\
\hline$\alpha$ And & 412 & $\gamma$ Cas & 408 \\
\hline$\alpha$ Aur (Capella) & 210,412 & $\gamma$ Per & 412 \\
\hline$\alpha$ Boo (Arcturus) & 274,407 & GSS 32 & 417 \\
\hline$\alpha \mathrm{CMa}$ (Sirius) & 140 & GSS 35 & 417 \\
\hline$\alpha \mathrm{CrB}$ & 414 & GU Tau & 417 \\
\hline$\alpha \mathrm{Equ}$ & 412 & На $1-16$ & 417 \\
\hline$\alpha$ Her & 386,396 & Haro $6-10$ & 417 \\
\hline$\alpha$ Lyr (Vega) & 297 & Her 36 & 416 \\
\hline$\alpha$ Ori (Betelgeuse) & $22,386,395$ & НH 1555 & 394 \\
\hline$\alpha$ Sco (Antares) & $328,386,396$ & HK Tau/A & 417 \\
\hline$\alpha^{1}$ Dra & 412 & HK Tau/G2 & 417 \\
\hline$\beta$ Ari & 412 & HR 1099 & 127 \\
\hline
\end{tabular}




\begin{tabular}{|c|c|c|c|}
\hline HR 8123 & 421 & $\mathrm{R}$ Aqr & 386,396 \\
\hline IK Tau & 335 & $\mathrm{R}$ Cas & 396 \\
\hline$\iota^{1} \mathrm{Lib}$ & 328 & R CVn & 396 \\
\hline IRC +10216 & 21 & R Cyg & 396 \\
\hline IRC+00198 & 377 & R Leo & 386 \\
\hline IRC +10013 & 377 & R Ser & 396 \\
\hline IRC +10216 & 386 & ROX 42B & 417 \\
\hline IRC+20034 & 377 & $\mathrm{RX} \mathrm{Cnc}$ & 76 \\
\hline IRC+ +20073 & 377 & $\mathrm{~S}$ Crb & 396 \\
\hline IRC +20190 & 377 & SAO 76140 & 76 \\
\hline IRC+20200 & 377 & Sgr A* & 379 \\
\hline IRC+30094 & 377 & $\sigma \mathrm{Sgr}$ & 328 \\
\hline IRC-10578 & 377 & SR 10 & 417 \\
\hline IRS 13 & 379 & SR 20 & 417 \\
\hline IRS 16 & 379 & SR 9 & 417 \\
\hline IRS 58 & 417 & Sun & $299,370,398$ \\
\hline IW Tau & 417 & T Cep & 396 \\
\hline Large Magellanic Cloud & 404 & T Tau & 391 \\
\hline Mars & 458,463 & Tau A & 245 \\
\hline Mercury & 461,465 & $\tau$ Per & 412 \\
\hline Moon & 460 & $\theta \mathrm{Aql}$ & 412 \\
\hline Mrk 501 & 125 & $\theta^{2} \mathrm{Tau}$ & 412 \\
\hline MSH 04-71 & 132 & Titan & 466 \\
\hline$\mu$ Сер & 396 & TX Cam & 127 \\
\hline NGC 1566 & 358 & U Her & 127 \\
\hline NGC 1672 & 358 & U Ori & 386 \\
\hline NGC 4151 & 344 & V Crb & 396 \\
\hline NGC 7552 & 355 & V CVn & 396 \\
\hline NML Tau & 386 & Vir A & 245 \\
\hline Nova Cygni 92 & 344,408 & VSS 35 & 417 \\
\hline NRAO 512 & 446 & VSS 42 & 417 \\
\hline ОН $127.8-0.0$ & 335 & VSSG 14 & 417 \\
\hline$o$ Ceti (Mira) & $24,386,395,408$ & VX Sgr & 386 \\
\hline$o$ Leo & 412 & VY CMa & 386 \\
\hline Orion nebula & 401 & W Aql & 386 \\
\hline$\phi \mathrm{Cyg}$ & 412 & X Oph & 396 \\
\hline$\phi$ Per & 408 & $\xi$ Cep A & 412 \\
\hline$\pi$ And & 412 & $\zeta$ Aur & 414 \\
\hline PKS 1830-211 & 132 & $\zeta$ Boo & 346 \\
\hline$\psi$ Per & 408 & $\zeta \mathrm{CMa}$ & 279 \\
\hline Q $0957+561$ & 454 & $\zeta \mathrm{Sgr}$ & 347 \\
\hline Q $1413+117$ & 454 & $\zeta \mathrm{Tau}$ & 408 \\
\hline Q $2237+0305$ & 453 & $\zeta^{1} \mathrm{Uma}$ & 412 \\
\hline $\mathrm{R} \mathrm{Aql}$ & 396 & -44275 & 412 \\
\hline
\end{tabular}

